\title{
Hominin evolution: A review of bipedal adaptations and cranial capacity as a means of studying evolution and analyzing speciation
}

\author{
Cassy Appelt*
}

\begin{abstract}
Hominin evolution and the field of paleoanthropology are cornerstones of paleontological inquiry. Much about hominin evolution has been learned since the discovery of the first Neanderthal specimen in 1856, but increasing ambiguity regarding the speciation of extinct hominins has created debate within the scientific community. A thorough look at the role of bipedal and cranial adaptations, selective pressures leading to their emergence, and the implications of their perseverance, has the capacity to clear up associated ambiguities in the field of paleoanthropology. Coupled with advances in technology, such as the accessibility of ancient DNA, it is possible to critically evaluate hominin morphology and its impact on reproductive barriers. As the field of paleoanthropology continues to inquire into our evolutionary past, a more concise and complete account of hominin evolution is possible.
\end{abstract}

Keywords: human evolution, Neanderthals, bipedalism, ancient DNA, molecular bioarchaeology, pelvis

\section{Introduction}

Hominin evolution, the limits of the fossil record and the speciation paradox

The topic of human evolution has been at the forefront of scientific inquiry since the Neander Valley discovery of Homo neanderthalensis (Neanderthal) remains in Germany in 1856 , leading to questions surrounding what exactly makes us human and how those criteria can be defended against the knowledge that we were not the only hominin species to have existed (de Miguel \& Henneberg
2001; Schmitz et al. 2002; Quintyn 2009). As the field of paleoanthropology evolves, its focus has shifted from striving to find reasons why we are unique to understanding how and when markedly "human" traits such as bipedalism and increased cranial capacity emerged (Foster, Raichlen \& Pontzer 2013). Since the discovery of Neanderthals in 1856, as many as six genera of extinct hominin species have been discovered and named (Quintyn 2009).

Divergence of the hominin clade from the genus Pan occurred before 7 million years ago (mya), distinguishing humans and their extinct ancestors within the family

\footnotetext{
*Department of Archaeology \& Anthropology, and the Department of Biology, College of Arts and Science, University of Saskatchewan, Saskatoon, SK, Canada Correspondence: cma392@mail.usask.ca
} 
Hominidae (deMonecal 2004; Zollikofer et al. 2005; Quintyn 2009). The term "hominin" refers to any species following this divergence, currently including members of the genus Homo, Australopithecus, Orrorin, Paranthropus, Ardipithecus, and Sahelanthropus (deMonecal 2004). The study of hominin evolution is relentlessly hindered by the absence of agreed methodology in identifying and establishing new hominin species, and a lack of understanding regarding modes of speciation as observed through fossilized remains (de Miguel \& Henneberg 2001; Schmitz et al. 2002; Quintyn 2009). Often, gaps in the fossil record are regarded as speciation events, leading to the explosion in the number of named hominin species and a trend towards "splitting" hominin remains into increasingly complex and possibly redundant categories rather than "lumping" fossils based on degrees of similarity (Lovejoy 2005a; Lovejoy 2005b; Quintyn 2009). The realm of paleoanthropology is surrounded by critiques regarding the designation of new hominin species as misunderstanding intraspecific variation rather than successfully identifying speciation events (de Miguel \& Henneberg 2001; Schmitz et al. 2002; Lovejoy 2005a, Lovejoy 2005b; Quintyn 2009). Perhaps the most significant challenge facing the study of human evolution is the medium of the study itself. Homo sapiens sapiens is the only extant hominin species, making the study of our evolutionary past based almost solely on the morphological comparison of fossil hominin remains (de Miguel \& Henneberg 2001; Schmitz et al. 2002; Quintyn 2009; Foster et al. 2013). Rarely are there instances in which a large sample of remains is available to provide a clear indication of our evolutionary history, or establish morphological speciation markers to clear up taxonomic uncertainties (de Miguel \& Henneberg 2001; Quintyn 2009).

The biological species model may be the most widespread and applicable definition in determining speciation, but presents a number of obstacles when studying extinct animals (Quintyn 2009). The model defines a species based on reproductive or naturally occurring barriers that isolate potentially interbreeding populations (Quintyn 2009). Both of these lines of evidence are inaccessible from static remains. In cases where it can be determined that hominin "species" existed within the same location and period of time, only those with harvestable DNA can be analyzed as to their genetic continuity (Relethford 2001; Quintyn 2009; Green et al. 2012; Hawks 2013). Such cases have demonstrated that our current species is a mosaic of genetic contributions from Neanderthals, Denisovans, and at least one as-yet unidentified hominin species (Relethford 2001; Green et al. 2012; Meyer et al. 2012; Hawks 2013; Bohlender, Yu, Huff \& Rogers 2016). Interbreeding between hominin species in the past seemingly satisfies the biological species model, even though morphology has been interpreted to indicate the opposite (Relethford 2001; Hawks 2013). This raises the question of at what point we can rely on morphological variation to designate species in specimens where harvestable DNA is not available.

Through the detailed study of hominin remains, it is possible to identify adaptive shifts in past populations, determine the prevalence of intraspecific and interspecific variation, and use this knowledge in approaching hominin speciation. The goal of this research paper is to explore what selective pressures drove morphological adaptations such as bipedalism and increased cranial capacity, and how these adaptations can be utilized to answer questions surrounding hominin speciation in general; of the most immediate concern in research today is the controversial question of humans interbreeding with Neanderthals. Paleoanthropology and the application of ancient DNA techniques have the capacity to offer a concise and informed look at what makes us unique as humans.

\section{Bipedalism as the earliest hominin adaptive trait}

The emergence of facultative bipedalism

It is a common misconception that bipedal walking in quadrupeds exhibits a significant decrease in the cost of locomotion and thus supported the transition to bipedal locomotion in the hominin lineage. In fact, there have been a number of skeletal adaptations that enable the transition from quadrupedal to fully bipedal locomotion over the course of hominin evolution (Sockol, Raichlen, \& Pontzer 2007; Raichlen, Pontzer, \& Sockol 2008; Foster et al. 2013). Based on measures of ground reaction force (GRF), which is the force generated through ground-foot contact and the resulting volume of muscles activated, bipedal locomotion in chimpanzees exhibits an increased energy cost. However, this increase $(10 \%)$ is not so significant that quadrupedal apes cannot utilize bipedalism habitually. The increase in GRF is primarily caused by the inability of quadrupeds to stand fully erect and extend their hip joints completely during swing phase of the walking cycle (Sockol et al. 2007; Raichlen et al. 2008; Foster et al. 2013). The walking cycle comprises stance phase, when the body is supported by the contact of both feet on the ground, and swing phase, when one foot is in contact with the ground and the other is lifted (Mann \& Hagy 1980). This inability to stand erect causes quadrupeds like chimpanzees to walk with a bent knee and bent hip (BKBH). Humans walking with $\mathrm{BKBH}$ also exhibit an associated energy cost from that of an erect posture, but this cost is lower still than the cost chimps exhibit (Sockol et al. 2007; Raichlen et al. 2008; Foster et al. 2013).

Energy costs associated with BKBH walking in quadrupedal apes revolves around increased flexion in the knee and hip joints as a consequence of the placement of the head and trunk (HAT) anterior to the pelvis (Lovejoy 2005a; Sockol et al. 2007; Raichlen et al. 2008; Foster et al. 2013). 
This anterior placement increases muscle activity in the erector spinae muscles, primary gluteals, and the hamstrings (Lovejoy 2005a; Lovejoy 2005b; Lovejoy 2007; Sockol et al. 2007; Raichlen et al. 2008; Foster et al. 2013). The decrease in cost associated with human BKBH walking surrounds the dynamics of the hip muscles. The dorsally located ischium and broadened pelvis in modern humans allows for $\mathrm{BKBH}$ walking without increased flexion at the hip, whereas the caudally located ischium and round pelvis in quadrupedal primates causes an increase (Lovejoy 2005a; Lovejoy 2005b; Sockol et al. 2007; Raichlen et al. 2008; Foster et al. 2013). The ischium is the site of a number of muscle attachments, including the hamstrings, which work antagonistically to the production of ground force reaction during hip extension. A caudally located ischium provides support to the HAT and pelvis during arboreal climbing, but leads to increased muscle activity during BKBH walking (Sockol et al. 2007; Raichlen et al. 2008; Foster et al. 2013). It is important to note that in obligatory bipeds such as modern humans, the hamstrings and other muscles are still an active part of the walking cycle, but BKBH walking does not increase this activity and increase the associated cost. Obligatory bipedalism is associated with a number of adaptations in the spine, pelvis, and knee that allow for significantly decreased energy costs in erect walking that have also reduced the cost of walking with $\mathrm{BKBH}$ compared to that of quadrupedal primates (Lovejoy 2005a; Lovejoy 2005b; Hooker 2007; Lovejoy 2007; Sockol et al. 2007; Raichlen et al. 2008; Foster et al 2013).

The Miocene (23.0-5.3 million years ago) in Africa is characterized by a warm and wet climate that continually became cooler and drier near the Pliocene (5-3-2.6 mya) boundary (deMenocal 2004; Isbell \& Young 1996). The hominin clade diverged from the genus Pan during the Late Miocene (Zollikofer et al. 2005). The significance of a drier environment is that the last common ancestor between the hominin and Pan line would have been faced with decreasing territory as the drying environment promoted the growth and dominance of open savannah, causing resources to become clumped and survival dependent on leaving the canopy to find food (Isbell \& Young 1996; deMonecal 2004; Carvalho et al. 2012). Any associated costs of BKBH bipedalism may have been outweighed by the advantage of having free hands to carry food and a heightened vantage to keep watch for predators (Isbell \& Young 1996; deMonecal 2004; Carvalho et al. 2012). Based on the analyses of BKBH locomotion in fully quadrupedal species, bipedalism does not carry an overwhelming energy demand. With the added benefits of transporting resources and spotting predators, the positive selection of traits that supported decreasing the cost associated with facultative bipedalism could have been favoured (Lovejoy 2005a; Lovejoy 2005b; Lovejoy 2007; Sockol et al. 2007; Raichlen et al. 2008; Carvalho et al. 2012; Foster et al. 2013).
The earliest indication of bipedalism in hominins is manifested in Sahelanthropus tchandenis, the earliest hominin discovered to date, as an inferior and anteriorly placed foramen magnum (Zindler 1978; Jablonski 1993; Zollikofer et al. 2005). While the postcranial remains of $S$. tchadensis have not been recovered for analysis regarding further evidence supporting bipedalism, the date of these remains coincides with the cooling and drying swing of the Late Miocene (Isbell \& Young 1996; deMonecal 2004; Zollikofer et al. 2005). The earliest hominin species with adequate postcranial remains, Ardipithecus ramidus, exhibits a similarly placed foramen magnum, as well as a caudally placed ischium like that of quadrupedal primates (Lovejoy 2005a; Lovejoy et al. 2009; White et al. 2009; White, Lovejoy, Asfaw, Carlson \& Suwa 2015). It has been hypothesized that the presence of an inward curve to the lumbar spine ("lordosis") and elongation of the hind limbs, in addition to a dorsally placed ischial tuberosity, would allow for proper HAT placement and reduced associated costs in BKBH walking (Lovejoy 2005a; Sockol et al. 2007). While Ar. ramidus remains do not exhibit the dorsal relocation of the ischial tuberosity, or any changes in the inferior pelvis to support efficient bipedalism, the presence of lumbar lordosis is apparent. In fact, Ar. ramidus remains have a mosaic of arboreal and bipedal adaptations. A caudally located ischial tuberosity, elongate inferior pelvis, and grasping hallux would have allowed it to navigate the canopy efficiently, while the lumbar lordosis of its spine, a mediolaterally widened superior pelvis, and elongated hind limbs (equal in length to the forelimbs, rather than shorter) would have provided the balance required for bipedalism at a lowered energy cost (Sockol et al. 2007; Lovejoy et al. 2009; White et al. 2009; White et al. 2015).

\section{Obligatory bipedalism emerged early in hominin evolution}

The emergence of completely erect walking and obligatory bipedalism was definitively established as early as 3.0 mya in Australopithecus africanus, an early descendent of Australopithecus afarensis. There is strong evidence that obligatory bipedalism may have been established 0.7 million years prior to that in Australopithecus afarensis, however adequate remains of this species' lumbar spine are fewer than Au. africanus (Lovejoy 2005; Lovejoy 2005b; Lovejoy 2007; Sockol et al. 2007; Raichlen et al. 2008; Foster et al. 2013). Australopithecus afarensis is regarded as the last common ancestor between the genus Paranthropus (or the robust Australopithecines) and the gracile Australopithecines. The "gracile Australopithecines" include species such as Australopthicus africanus and Australopithecus garhi, considered to be the ancestor to the genus Homo (Hunt 1994; Asfaw et al. 1999; Lovejoy 2005a; Lovejoy 2005b). Australopithecus afarensis corresponds to the increasingly arid and cool climate of the early Pleistocene 
(beginning $5.3 \mathrm{mya}$ ), and Au. africanus corresponds to the peak of aridity of the Mid-Pleistocene (3.2-2.6 mya), providing further selective pressure to be able to walk efficiently between clumped resources (deMonecal 2004).

As discussed, adaptive traits allowing for the emergence of a fully erect stature are necessary to reduce energy costs associated with bipedalism. Adaptations in the pelvis, knee, and lower spine directly influence HAT placement relative to the pelvis. In quadrupeds the HAT is anterior to the pelvis, but the body is supported on either end of the trunk and the weight of the trunk itself is shared between them. However, during bipedal locomotion there is only one support (the pelvis) and anterior placement of the trunk causes strain on muscles such as hamstrings and the primary gluteals (Lovejoy 2005a; Lovejoy 2005b; Sockol et al. 2007; Raichlen et al. 2008; Foster et al. 2013). The primary gluteals (gluteus maximus, medius, and minimus) are associated with parts of the pelvis (ilia) and function to stabilize the trunk. Particularly important to bipedal locomotion, the gluteus maximus functions to keep the pelvis from pitching forward (Lovejoy 2005a; Lovejoy 2005b). The hamstrings are associated with both the pelvis and the knee, working to stabilize the trunk during knee extension (Lovejoy 2007; Sockol et al. 2007). These muscles are influenced by the movement of the HAT directly above the point of impact with the ground, leading to optimal positioning and functioning of each muscle group (Lovejoy 2005a; Lovejoy 2005b; Sockol et al. 2007; Raichlen et al. 2008; Whitcome 2012; Foster et al. 2013). Walking in a BKBH manner may not be associated with substantial energy costs, but the resulting fatigue is not compatible with prolonged locomotion (Lovejoy 2005a; Lovejoy 2005b). As a result, $\mathrm{BKBH}$ bipedalism does not persist in the transition from an arboreal to a terrestrial existence (Lovejoy 2005a; Lovejoy 2005b; Sockol et al. 2007; Foster et al. 2013).

Transverse expansion of the pelvis is a trait supporting facultative bipedalism present in Ar. ramidus remains. This expansion is necessary to increase the space around the lumbar/sacral joint to provide the lumbar flexibility required for lordosis, and as such, this trait persists in Australopithecine remains (Lovejoy 2005a; Lovejoy et al. 2009; Whitcome 2012). Additionally, Australopithecines have a broadened and shortened sacrum that further increases lumbar-sacral joint space, and broader ilia when compared to earlier hominin pelvises (Haeusler, Martelli \& Boeni 2002; Lovejoy 2005a; Whitcome 2012). Widening of the superior pelvis, particularly the ilia, indicates the expansion and relocation of the gluteus maximus, gluteus medius, and gluteus minimus muscles such that they effectively stabilize the trunk during the walking cycle (Lovejoy 2005a; Lovejoy 2005b; Whitcome 2012). This changes the weight loading dynamics of the frame by decreasing the weight loaded onto the femur. Reduction in the amount of cortical bone of the superior femoral neck from that of quadrupeds (which exhibit complete ring of cortical bone) is exhibited in Au. africanus remains, but not in Au. afarensis (Lovejoy 2005a; Lovejoy 2005b; Lovejoy 2007). In regards to the inferior pelvis, Au. afarensis and descendent Australopithecines exhibit a dorsal orientation of the ischial tuberosity. Dorsal relocation of the ischial tuberosity allows the hamstrings (biceps femoris, semimembranosus, and semitendinosus muscles) that originate here to function efficiently and allow both the hip and knee to extend fully when the leg is raised (Sockol et al. 2007).

The knee also exhibits a range of changes from that of a quadrupedal primate's knee. The mammalian knee joint is very weak due to the nature of the joint itself, with femoral condyles balanced on a shallow tibial concavity and stabilized only by a musculotendonous sheath and four ligaments (Lovejoy 2007). In Au. afarensis and Au. africanus, the opposability of the hallux is lost and flexibility of the tarsus/metatarsus joints used to propel the step is reduced (Lovejoy 2007; Raichlen et al. 2008). This is compensated by separation of the tibia and patella to allow for increased range of motion of the knee joint, providing effective propulsion and limb extension. However, this joint is naturally under a considerable amount of stress which only increases in the shift to bipedalism (Lovejoy 2007). In quadrupeds, the primary source of joint stress is from the action of the single quadriceps femoris muscle group, which is flexed during the entire walking cycle (Lovejoy 2007; Sockol et al. 2007; Foster et al. 2013). The quadriceps muscles compress the patella against femur, preventing dislocation of the joint and supporting the limb (Lovejoy 2007). Bipeds are considered to be tibia-dominant, as the main source of joint stress comes from tibia-femur compression during limb extension and stress generated from the weight of the HAT during swing phase. As a result, Australopithecines and later hominins display a high lateral patellar lip of the femur to prevent knee dislocation and facilitate the retention of the patella by the quadriceps femoris muscle group during heelstrike (Lovejoy 2007). Bipeds also exhibit an elliptically shaped distal femur and proximal tibia due to the prolonged contact between the epiphyses during the walking cycle (Lovejoy 2007). In particular, the anterior/posterior expansion of the lateral epicondyle and the increased meniscal axes of the distal femur increase the available surface area for cartilage, allowing for effective weight transmission across the joint at a distinct bicondular angle only achievable by obligatory bipedalism (Lovejoy 2007).

Adaptations in the lower spine aid in the movement of the HAT above the pelvis. Dorsal wedging of lumbar centra indicate the presence of lordosis of the lower spine of Au. africanus, which allows the center of mass to be positioned directly above the pelvis (Lovejoy 2005a; Lovejoy 2005b; Whitcome 2012). This eliminates excess strain placed on the musculature of the hips and spine to keep the trunk from pitching forward, and centers the weight evenly on 
either side of the spine to avoid differential stress and possible injury (Lovejoy 2005a; Whitcome 2012). The presence of wedged centra in the lordotic curve spreads the forces acting on the vertebrae in a coplanar manner (in the same plane) rather than subjecting them to compressive force (spreading inwards) that causes compression injuries (Lovejoy 2005a; Whitcome 2012). The curve of the lumbar spine itself allows for increased load carrying capacity as inflection points along the vertical axis (between kyphosis of the thoracic spine and lordosis of the lumbar spine) are close together, decreasing their functional length and increasing the carrying capacity (Whitcome 2012). As a result, Au. afarensis and $A U$. africanus exhibit a dorsal relocation of erector spinae muscle attachments to the vertebral pedicles from the vertebral centra as movement of the HAT leads to a reduction in their activity. The erector spinae muscles span the vertebral column and function to keep the trunk erect (Haeusler et al. 2002; Lovejoy 2005a; Whitcome 2012). Further, Au. africanus exhibits coronal vertebral facet orientation, which supports increased spinal flexibility and prevents injury during walking (Lovejoy 2005a; Whitcome 2012). These traits, such as dorsal erector spinae muscle attachments and coronal-oriented vertebral facets, are exhibited in Au. afarensis remains, however the lumbar spine remains of this species are incomplete and offer less information regarding the definitive establishment of traits supporting obligatory bipedalism (Lovejoy 2005b).

The spinal flexibility and lumbar lordosis definitively established in Au. africanus, and potentially in Au. afarensis, indicate the complete abandonment of arboreal life (Lovejoy 2005a; Lovejoy 2005b). Adaptations to an arboreal mode of life include a restricted length of the lumbar spine and sagittal orientation of vertebral facets to create a stable, non-mobile spine (Haeusler et al. 2002; Lovejoy 2005a; Lovejoy 2005b; Whitcome 2012). Short-backed apes (arboreal primates) have a pattern of reduction in the number of vertebrae in the spine to increase stability and minimize injury (Lovejoy 2005a). While it is debated whether Au. africanus had five or six true lumbar vertebrae (modern humans have five lumbar vertebrae) it is unchallenged that the six lowest pre-sacral vertebrae in the spinal sequence functioned in the lumbar lordosis. The presence of dorsal wedging and the coronal facet orientation indicate that they would have been a part of the lumbar lordosis and thus supported a flexible lumbar spine beyond the abilities of modern humans (Haeusler et al. 2002; Lovejoy 2005a; Lovejoy 2005b; Whitcome 2012). Increased spinal flexibility would have been biomechanically advantageous due to the added weight of their long forelimbs, which are remnant arboreal adaptations and later reduced in the genus Homo (Lovejoy 2005a; Lovejoy 2005b; Whitcome 2012). Further, this increased spinal flexibility is significant as it could not have emerged if the species were arboreal, as it holds no adaptive benefit (Lovejoy 2005a; Lovejoy 2005b). Increasing the number of lumbar vertebrae present in the lumbar spine leads to an increased risk of spinal injury, and as such a reduction from six to five lumbar vertebrae occurred after the Australopithecines (Haeusler et al. 2002; Lovejoy 2005a; Whitcome 2012). The incidence of spinal injuries and spinal deformations such as scoliosis in modern humans still greatly exceeds the incidence in other primates, a trade-off between optimal lordosis and the risk of developmental deformations (Haeusler et al. 2002; Lovejoy 2005a).

\section{Increased cranial capacity in later hominins}

Much like the shift to bipedalism, the trend towards increased cranial capacity is thought to be influenced by the changing climate of the Pleistocene. As previously discussed, the Pleistocene epoch was a time during which there was a peak in cool and arid climate approximately 3.22.6 mya (deMonecal 2004). This period of time coincides with the emergence of the genus Homo, specifically Homo habilis 2.5 mya, and an increase in cranial capacity to 503$661 \mathrm{~mL}$ (or cc; volume measure) compared to that of the 450cc of Australopithecus africanus (Zindler 1978; Ruff 1991; Kappelman 1996; Liebermann, McBratney \& Krovitz, 2002; deMonecal 2004). There is evidence for dietary changes prior to the emergence of Homo that provided the high caloric diet, rich in proteins and fats, required to support the growth and maintenance of expensive brain tissue (Varki 2001; McPherron et al. 2010; Varki 2010; Hublin, Neubauer \& Gunz 2015). Evidence of stone tool production and use as early as 3.3 mya attributed to Au. africanus suggests that meat was a staple part of their diet, providing the caloric backdrop necessary for the emergence of a large brain in Homo (Zindler 1978; Ruff 1991; deMonecal 2004; McPherron et al. 2010).

Continuing in the hominin lineage is a pattern of accelerated cranial capacity occurring after Homo habilis. Next in sequence, early Homo erectus crania (1.8-1.5mya) measure goocc. Following the expansion out of Africa, Homo erectus cranial capacities increase to as much as $1000 \mathrm{cc}$ in Asia and up to $1200 \mathrm{cc}$ in Europe. The latest hominins include Homo neanderthalensis (230,000-30,000 years ago), which have been estimated to have capacities anywhere from $1500 \mathrm{cc}-1750 \mathrm{cc}$, and Homo sapiens (direct ancestors to the modern human population, Homo sapiens sapiens) with cranial capacities $1250 c c-1400 c c$ in measure (Bruner 2007; Meyer et al. 2012; Rightmire 2013; Hublin et al. 2015).

\section{Increased cranial capacity is related to maternal pelvis size}

The pattern of increased cranial capacity in hominin evolution has more implications than the potential for increased intelligence. Birthing large brained infants is undeniably tied to obstetric complications and increased maternal mortality (Robillard, Chaline, Chaouat \& Hulsey 
2003; Whitcome, Shapiro \& Lieverman 2007; Wittman \& Wall 2007; Fransicus 2009; DeSilva 2011; O'Connell \& DeSilva 2013; Hublin et al. 2015). Modern human infants weigh as much as $6 \%$ of their mother's mass, exceeding the expected correlation by more than $50 \%$ (catarrhine maternal/infant weight correlation) and leading to trade-offs with birth complications, biomechanical challenges, and maternal metabolic constraints (Whitcome et al. 2007; Wittman \& Wall 2007; Franciscus 2009; DeSilva 2011; Dunsworth, Warrener, Deacon, Ellison \& Pontzer 2012).

Maternal adaptations for the increased cranial size of infants include a number of sexually dimorphic traits in the pelvis. Human females have ilia that are wider and exhibit wider sciatic notches, a rounder pelvic inlet that is coupled with a flat and broad sacrum to maximize the outlet's area, and a higher number of dorsally wedged vertebrae such that females can further extend the arch in their back to carry their center of mass above their pelvis during later terms of pregnancy (Robillard et al. 2003; Whitcome et al. 2007; Wittman \& Wall 2007; Franciscus 2009; DeSilva 2011). Such adaptations are thought to conflict with bipedal biomechanics (Robillard et al. 2003; Lovejoy 2005; Lovejoy 2005; Whitcome et al. 2007; Wittman \& Wall 2007; Franciscus 2009; DeSilva 2011). Functional bipedalism constrains the width of the pelvis, leading to a conflict between selective pressures to increase pelvic dimensions for a large infant cranium, and to constrain the pelvis within optimal size for bipedal mechanisms (Whitcome et al. 2007; Wittman \& Wall 2007; Franciscus 2009; DeSilva 2011). As only half of the population would benefit from a wider pelvis beyond that which is necessary for efficient bipedalism, there is a balance between selective pressures to both constrain and widen the pelvis. While it is important to understand the dynamics surrounding the maternal pelvis and constraints on its size relative to its function, maternal metabolic cost is a key factor that influences gestation length and fetal growth maximums (Dunsworth et al. 2012; Hublin et al. 2015). Human females expend up to twice their basal metabolic rate (BMR) by the sixth month of pregnancy, overlapping with the maximum sustained metabolic rate in humans (2.0-2.5X BMR). By the $36^{\text {th }}-40^{\text {th }}$ week of pregnancy, fetal energy demands surpass that which is sustainable by the mother, and it is around this peak that labour takes place. While fetuses with larger crania and an increased capacity for cognition are advantageous, it is the energy cost to the mother that ultimately constrains fetal growth prior to delivery (Dunsworth et al. 2012).

With the emergence of the genus Homo there is a general increase in body size, including increases in pelvic dimensions (Bruner 2007; Simpson et al. 2008). An increase in the size of the pelvis naturally allows for a larger cranium to pass through the birth canal (Simpson et al. 2008; Weaver \& Hublin 2009). Within the Homo genus, there is an increase in cranial capacity from approximately $550 \mathrm{cc}$ in Homo habilis to goocc in Homo erectus (Bruner 2007; Simpson et al. 2008). This is a substantial increase considering the pelvic dimensions of Homo erectus do not show additional signs of obstetric specialization for birthing large brained infants (Simpson et al. 2008; Weaver \& Hublin et al. 2009). Based on measurements of the pelvis, it is possible that the transversally wide pelvis of Homo erectus could have passed a fetal skull as large as 230cc, establishing an emergent pattern of secondary altriciality, the birth of helpless infants combined with accelerated development over an extended juvenile period (Wittman \& Wall 2007; Simpson et al. 2008; Franciscus 2009; DeSilva 2011).

Secondary altriciality accounts for the exaggerated encephalization of later hominins

As the cost and benefit of lengthened gestation and increased cranial capacity of altricial neonates is maximized and balanced with constraints on maternal metabolism and the maternal pelvis, there is a change in the pattern of growth of hominin young. In general, primate pelvises are round and the head size of their infants does not exceed the space of the birth canal; the heads and shoulders of their young can pass through the birth canal without assistance, and there is no prevalence of obstetric complication in primates other than humans (Wittman \& Wall 2007; Simpson et al. 2008; Weaver \& Hublin 2009; DeSilva 2011; Dunsworth et al. 2012). It has been estimated that Homo habilis and Homo erectus could have given birth to young with a maximum cranial capacity of 230cc, and yet the actual cranial capacity of both these species is considerably larger (Kappelman 1996; Lieberman et al. 2002; Simpson et al. 2008; Weaver \& Hublin 2009; Hublin et al. 2015). Rather than relying on increased encephalization of fetuses, which would cause a significant increase in maternal cost, the emphasis on secondary altriciality and accelerated brain development in juveniles becomes a pattern (Lieberman 2002; O'Connell \& DeSilva 2013). Positive selective pressure placed on juveniles to compensate for increased costs associated with brain tissue development and longer juvenile periods would have included the adaptive benefit of increased cognitive capabilities and the transmission of cultural practises (deMonecal 2004; Bruner 2007; McPherron et al. 2010; O'Connell \& DeSilva 2013).

An increased reliance on secondary altriciality is definitively demonstrated as early as Homo erectus (Simpson et al. 2008; Weaver \& Hublin 2009; O'Connell \& DeSilva 2013). The remains of a one-year-old Homo erectus child's calvarium indicates that, at one year of age, the child had achieved $72-84 \%$ of the adult cranial capacity (Simpson et al. 2008; O'Connell \& DeSilva 2013). Modern humans show an even more intense rate of secondary altriciality as a comparative level of brain development and growth is achieved in human juveniles after the age of four. The advantage of a longer maturation and juvenile period is that 
development is the optimal time for learning and enculturation. The longer the brain develops, the more opportunity there is for cultural transmission and learning, and the more complex the culture can become (Dunsworth et al. 2012; Hublin et al. 2015).

Homo erectus not only shows a significant increase in cranial capacity reliant on secondary altriciality, but is the first hominin species to have moved out of Africa and into parts of Europe and Asia (Bruner 2007; Simpson et al. 2008; Rightmire 2013). From Homo erectus, there are a number of hominin lineages that diverge in different parts of Eurasia and from remaining groups in Africa (Bruner 2007). While there are debates about the emergence of Homo heidelbergensis, which may have given rise to Homo florensiensis, Homo altai, Homo neanderthalensis, and Homo sapiens (ancestral to modern humans, Homo sapiens sapiens), there is little disagreement surrounding the rapid increase in cranial capacity in hominins following the movement of Homo erectus out of Africa (Kappelman 1996; Bruner 2007; Rightmire 2013).

Increased dependence of secondary altriciality offers a likely mechanism for such exaggerated patterns of encephalization in hominins in the last 500,000 years, but Homo sapiens exhibit further obstetric specialization that allow for fetal cranial capacity to surpass pelvic dimensional constraints. An anteroposterior widening of the pelvis in Homo sapiens allows the birthing process itself to change, resulting in the passage of a larger crania than that which is compatible with dimensions of the birth canal (Bruner 2007; Wittman \& Wall 2007; Franciscus 2009; Weaver \& Hublin 2009; DeSilva 2011). Rotational birth consists of the infant twisting such that the widest part of its head aligns with the widest part of the maternal pelvis as it moves through the birth canal. The infant descends from the womb into the birth canal with its head facing down and to the side such that the sagittal diameter of its head engages the pelvis transversally. Partway through the birth canal, the infant twists so that it faces more posteriorly and the sagittal plane is aligned with the pelvic axis. The head of the infant emerges from the birth canal facing the posterior, and here the infant twists again such that the shoulders and body can emerge underneath the pubis (Wittman \& Wall 2007; Franciscus 2009). In other primates, there is no rotation as the infant's head is smaller than the pelvic outlet (Wittman \& Wall 2007; Bruner 2007). In hominins with an oval shaped pelvis, the head of the infant would have descended into the birth canal transversally oriented, and rotation would have occurred only after the head emerged from the pelvic outlet to align the shoulders and allow the body to emerge (Lovejoy 2005a; Lovejoy 2005b; Bruner 2007; Franciscus 2009).

Beyond morphological assessment: The role of ancient DNA in understanding hominin evolution
Cranial capacity is an important pattern in hominin evolution due to its implications for cognitive ability. Beyond the analysis of cranial volume, endocasts and endocranial impressions have been used to highlight and study differences between modern humans and extinct hominins (Bruner 2007; Boas 2012; McPherron et al. 2010). For instance, Neanderthals exhibit an increased frontal region and reduced parietal regions of the brain compared to that of modern humans. Significantly different from Neanderthals, Homo sapiens exhibit globularization and increased parietal regions of the brain. The implications of these differences regarding inter-hemispheric interaction and cognitive abilities are not well understood (Brunner 2007; Boas 2012). The parietal lobes are linked to visualspatial integration, tool use, and generation of inner reality. This has been taken in the past to support cultural differences between anatomically modern humans and Neanderthals (Bruner 2007). On the other hand, Neanderthals exhibited increased frontal region, which is associated with motor skills, long term memory, and language, all things that are considered to be well developed in modern humans (Brunner 2007; Boas 2012). Based on endocasts and endocranial impressions, assumption about the differences between modern humans and extinct hominins, such as Neanderthals, can be misleading.

With the development of DNA extraction and amplification technology, the potential to analyze DNA from archaeological sites has become a reality. In such cases, nucleic acid decay and damage place constraints on the ability to successfully amplify genetic material from hominin remains (Relethford 2001; Green et al. 2012; Meyer et al. 2012; Hawks 2013). Ancient DNA (aDNA) techniques are theoretically limited to specimens younger than 1,000,000 years in age based on these factors (Green et al. 2012; Meyers et al. 2012; Hawks 2013). These techniques have proved advantageous in studying hominins, a part of our immediate evolutionary past, providing evidence that hominins ancestral to modern human populations interbred with Neanderthals, Denisovans, and possibly other hominin species (Green et al. 2012; Meyers et al. 2012; Hawks 2013; Bohlender et al. 2016).

In particular, the Neanderthal genome has been the focus of much attention and research. Genomic DNA studies indicate that, while humans exhibit $0.10 \%$ genetic differences within the modern population, there is only an approximate $0.15 \%$ difference between modern human genomes and that of the Neanderthals (Hawks 2013). Further analysis indicates that, of this $0.15 \%$ difference, modern humans of non-African descent can be up to $4 \%$ more similar genetically to Neanderthals than those of African descent (Green et al. 2012; Meyer et al. 2012; Hawks 2013; Vernot \& Akey 2015). This indicates that, while the divergence of Homo neanderthalensis from Homo sapiens occurred between $270,000-440,000$ years ago, at least one 
instance of secondary contact and interbreeding took place before Eurasian haplotypes diverged from each other (Green et al. 2012; Hawks 2013). This places the last instance of genetic flow between Neanderthals and the ancestors of modern Eurasians approximately 37,000-82,000 years ago, at which point anatomically modern humans had emerged and were considered to be Homo sapiens (Green et al. 2012; Hawks 2013). Additionally, recent work suggests that people of East Asian descent can have up to $20 \%$ more Neanderthal DNA in their genome when compared to others of nonAfrican descent (Vernot \& Akey 2015; Kim \& Lohmueller 2015; Mendez et al. 2016). These genetic differences are not well understood. However, genetic drift, population fluctuations, and the effects of weakly deleterious alleles entering the human gene pool are implicated (Vernot \& Akey 2015; Kim \& Lohmueller 2015; Mendez et al. 2016).

Mitochondrial DNA (mtDNA) and Y-chromosomal DNA analysis indicates a very low genetic continuity between modern human populations and Neanderthals (Relethford 2001; Green et al. 2012; Hawks 2013; Vernot \& Akey 2015; Kim \& Lohmueller 2015; Mendez, Poznik, Castellano \& Bustamante 2016). This low genetic continuity has been used in the past to fuel debates and refute instances of interbreeding between Homo neanderthalensis and Homo sapiens. As both mtDNA and the Y-chromosome are inherited unilinearly, this could indicate that representatives of the maternal and paternal haplotypes present in Neanderthal populations left the modern human population by chance in a process called genetic drift (Relethford 2001; Hawks 2013).

With regard to mtDNA, the morphological assessment of hominin remains in regard to bipedalism and cranial capacity increases may provide another explanation as to the low genetic continuity between Neanderthals and modern human populations. The current fossil record indicates that Homo sapiens (and by extension, modern humans) are the only hominin species to have an anteroposteriorly widened pelvis (Franciscus 2009; Weaver \& Hublin 2009; DeSilva 2011). This, along with a unique pattern of rotational birth, compensates for the birth of an infant with a larger head size than compatible with pelvic dimensions (Franciscus 2009; Weaver \& Hublin 2009; DeSilva 2011). Based on the role of an anteroposteriorly wide pelvis in birthing infants with crania exceeding pelvic dimensions, the oval shaped pelvis of female Neanderthals may have acted as a partial reproductive barrier leading to a differential genetic contribution in the Homo sapiens sapiens gene pool. This would account for the biased representation of Neanderthal maternal continuity in modern human populations today, and provide an alternative or additional hypothesis regarding the lack of mtDNA continuity to that of genetic drift.

However, Y-chromosome genetic continuity is also low between modern humans and Neanderthals. In fact, recent research indicates that the $\mathrm{Y}$-chromosome haplotype present in an $\sim 120 \mathrm{~kb}$ sequenced exome of one Neanderthal specimen in El Sidron, Spain ( 49,000 ya) is extinct, showing no closer similarity to people of African or Eurasian descent (Mendez et al. 2016). One possible explanation is a blanketed assignment of genetic drift causing this particular haplotype to disappear from modern human populations. However, it has been suggested that Neanderthal DNA in modern human populations has been subjected to purification selection. Some alleles that are Neanderthal in origin are weakly deleterious in human populations, whereas they would have been selectively neutral in the small Neanderthal population around the time interbreeding between the two populations is suggested to have occurred (Kim \& Lohmueller 2015). As a result, in large human populations such deleterious alleles are often removed from the gene pool by natural selection (Kim \& Lohmueller 2015; Vernot \& Akey 2015). This could explain the higher prevalence of Neanderthal DNA in modern East Asian populations, as historically this area had small populations and were susceptible to genetic bottlenecks (Kim \& Lohmueller 2015). Examples of deleterious alleles from Neanderthal populations entering the human population include those associated with histocompatibility genes and genes that are implicated in male Y-chromosome infertility (Kim \& Lohmueller 2015; Mendez et al. 2016). The Neanderthal Ychromosome in the El Sidron, Spain, specimen carries mutations in genes that influence spermatogenesis, which could potentially reduce fertility, and histocompatibility alleles, which might have contributed to secondary recurrent miscarriages (Mendez et al. 2016). Such alleles may have caused difficulty in successful breeding between Neanderthal males and human females, and causing male offspring carrying this Y-chromosome haplotype to have difficulty fathering children. This may explain why, once Neanderthal DNA entered human populations, the Ychromosome haplotype did not persist in the human gene pool. These factors, along with those influencing female Neanderthal contributions to the modern human gene pool, may have contributed to partial reproductive barriers between Neanderthals and humans. However, as suggested by current aDNA studies, complete reproductive isolation and thus true speciation between these two populations would not have been established prior to secondary contact and genetic admixture $38,000-82,000$ years ago.

\section{Conclusion}

The field of paleoanthropology has been characterized in the past by ambiguity and unrest as the scientific community approached concepts such as the identification of interspecific and intraspecific variation with the goal of understanding speciation of extinct hominins (Quintyn 2009). What can only be considered an explosion of misinterpreted speciation events, leading to a complex and

University of Saskatchewan Undegraduate Research Journal 
often redundant network of hominin species, has the potential to be resolved through careful morphological study (de Miguel \& Henneberg 2001; Schmitz et al. 2002; Lovejoy 2005a). As the fossil record regarding hominin evolution becomes more complete, the potential to understand the sequence and events in hominin evolutionary history, such as the emergence of bipedalism and increased cranial capacity, becomes possible. The benefit of continued research as modern technology evolves is that it provides a method for answering questions about speciation in extinct hominin species. A complete understanding about the selective pressures and circumstances that drove the emergence of bipedalism and increased cranial capacity not only provides a concise representation of hominin evolution as it is understood today, but it also has the potential to resolve larger issues within the field of paleoanthropology. This is complemented by the role of ancient DNA in furthering the study of partial reproductive barriers between Homo neanderthalensis and Homo sapiens. There is still much to discover regarding hominin evolution as paleoanthropology continues to discover more hominin fossil representations, and as modern technology itself evolves. 


\section{References}

Asfaw, B., White, T. D., Lovejoy, O., Latimer, B., Simpson, S., \& Suwa, G. 1999. Australopithecus garhi: a new species of early hominid from Ethiopia. Nature, 371, 306-312. doi:10.1038/371306ao

Bruner, E. 2007. Cranial shape and size variation in human evolution: structural and functional perspectives. Childs Nervous System, 23, 1357-1365.

Boas, M. 2012. Hominin endocasts topography: an analysis using geographic information systems (Master's thesis). Boca Raton, FL: Florida Atlantic University.

Bohlender, R., Yu, Y., Huff, C., \& Rogers, A. 2016. PgmNr 73: A complex history of archaic admixture in modern humans [Presentation]. Presented at the Annual Meeting of the American Society of Human Genetics, Vancouver, BC.

Carvalho, S., Biro, D., Cunha, E., Hockings, K., Mcgrew, W. C., Richmond, B. G., \& Matsuzawa, T. 2012. Chimpanzee carrying behaviour and the origins of human bipedality. Current Biology, 22, 180-181. doi:10.1016/j.cub.2012.01.052

de Miguel, C., \& Henneberge, M. 2001. Variation in hominid brain size: how much is due to method? Homo, 52, $3-58$.

deMonecal, P. B. 2004. African climate change and faunal evolution during the Pliocene-Pleistocene. Earth and Planetary Science Letters, 220, 3-24.

DeSilva, J. M. 2011. A shift toward birthing relatively large infants early in human evolution. Proceedings of the National Academy of Science, 108, 1022-1027.

Dunsworth, H. M., Warrener, A. G., Deacon, T., Ellison, P. T., \& Pontzer, H. 2012. Metabolic hypothesis for human altriciality. Proceedings of the National Academy of Sciences, 109, 15212-15216. doi:10.1073/pnas.1205282109

Foster, A. D., Raichlen, D. A., \& Pontzer, H. 2013. Muscle force production during bent-knee, bent-hip walking in humans. Journal of Human Evolution, 65, 294-302.

Franciscus, R. G. 2009. When did the modern human pattern of childbirth arise? New insights from an older Neandertal pelvis. Proceedings of the National Academy of Science, 23, 9125-9126.

Green, R. E., Krause, J., Briggs, A. W., Maricic, T., Stenzel, U., Kircher, M., et al. 2012. A draft sequence of the Neandertal genome. Science, 328, 710-722.
Haeusler, M., Martelli, S. A., \& Boeni, T. 2002. Vertebrae numbers of the early hominid lumbar spine. Journal of Human Evolution, 43, 621-643.

Hawks, J. 2013. Significance of Neandertal and Denisovan genomes in human evolution. Annual Review of Anthropology, 42, 433-449.

Hooker, J. J. 2007. Bipedal browsing adaptations of the unusual late Eocene-earliest Oligocene tylopod Anoplotherium (Artiodactyla, Mammalia). Zoological Journal of the Linnaen Society, 151, 609659.

Hublin, J. J., Neubauer, S., \& Gunz, P. 2015. Brain ontogeny and life history in Pleistocene hominins. Philosophical Transactions Royal Society, 370, 20140062. doi:10.1098/rstb.2014.0062

Hunt, K. D. 1994. The evolution of human bipedality, ecology and functional morphology. Journal of Human Evolution, 26, 183-202.

Isbell, L. A., \& Young, T. P. 1996. The evolution of bipedalism in hominids and reduced group size in chimpanzees: alternative responses to decreasing resource availability. Journal of Human Evolution, 30, 389-397.

Jablonski, N. G. 1993. Origin of habitual terrestrial bipedalism in the ancestor of the Hominidae. Journal of Human Evolution, 24, 259-280.

Kappelman, J. 1996. The evolution of mass and relative brain size in fossil hominids. Journal of Human Evolution, 30, 243-276.

Kim, B. Y., \& Lohmueller, K. E. 2015. Selection and reduced population size cannot explain higher amounts of Neandertal ancestry in East Asian than in European human populations. American Journal of Human Genetics, 96, 454-461. doi:10.1016/j.ajhg.2014.12.029

Lieberman, D. E., McBratney, B. M., \& Krovitz, G. 2002. The evolution and development of cranial form in Homo sapiens. Proceedings of the National Academy of Science, 99, 1134-1139.

Lovejoy, C. O. 2005a. The natural history of human gait and posture: Part 1. Spine and pelvis. Gait and Posture, 21, 95-112.

Lovejoy, C. O. 2005b. The natural history of human gait and posture: Part 2. Hip and thigh. Gait and Posture, 21, $113-124$.

Lovejoy, C. O. 2007. The natural history of human gait and posture: Part 3. The knee. Gait and Posture, 25, 325-341. 
Mann, R. G. \& Hagy, J. 1980. Biomechanics of walking, running, and sprinting. The American Journal of Sports Medicine, 8, 345-350.

McPherron, S. P., Alemseged, Z., Marean, C. W., Wynn, J. G., Reed, D., Geraads, D. et al. 2010. Evidence for stone-tool-assisted consumption of animal tissues before 3.39 million years ago at Dikka, Ethiopia. Nature, 466, 857-86o.

Mendez, F. L., Poznik, G. D., Castellano, S., \& Bustamante, C. D. 2016. The divergence of Neandertal and modern human y chromosomes. American Journal of Human Genetics, 98, 728-734.

doi:10.1016/j.ajhg.2016.02.023

Meyer, M., Kircher, M., Gansuage, M. T., Li, H., Racimo, F., Mallick, S., et al. 2012. A high-coverage genome sequence from an archaic Denisovan individual. Science, 388, 222-226.

O'Connell, C. A., \& DeSilva, J. M. 2013. Mojokerto revisited: evidence for an intermediate pattern of brain growth in Homo erectus. Journal of Human Evolution, 65, 156-161.

Quintyn, C. 2009. The naming of new species in hominin evolution: a radical proposal-a temporary cessation in assigning new names. Journal of Comparative Human Biology, 60, 307-341.

Raichlen, D. A., Pontzer, H., \& Sockol, M. 2008. The Laetoli footprints and early hominin locomotor kinematics. Journal of Human Evolution, 54, 112117.

Rightmire, P. G. 2013. Homo erectus and Middle Pleistocene hominins: brain size, skull form, and species recognition. Journal of Human Evolution, 65, 223252.

Relethford, J. H. 2001. Absence of regional affinities of Neandertal DNA with living humans does not reject multiregional evolution. American Journal of Physical Anthropology, 115, 95-98.

Robillard, P., Chaline, J., Chaouat G., \& Hulsey, T. C. 2003. Preeclampsia/eclampsia and the evolution of the human brain. Current Anthropology, 44, 130-135.

Ruff, C. B. 1991. Climate and body shape in hominid evolution. Journal of Human Evolution, 21, 81-105.

Schmitz, R. W., Serre, D., Bonani, G., Feine, S., Hillgruber, F., Krainitzki, H., et al. 2002. The Neandertal type site revisited: interdisciplinary investigations of skeletal remains from the Neander Valley, Germany. Proceedings of the National Academy of Science, 99, 13342-13347.
Simpson, S. W., Quade, J., Levin, N. E., Butler, R., DupontNivet, G., Everett, M., et al. 2008. A female Homo erectus pelvis from Gona, Ethiopia. Science, 322, 1089-1092.

Sockol, M. D., Raichlen, D. A., \& Pontzer, H. 2007. Chimpanzee locomotor energetics and the origin of human bipedalism. Proceedings of the National Academy of Science, 30, 12265-12269.

Varki, A. 2001. Loss of N-glycolyneuraminic acid in humans: mechanisms, consequences, and implications for hominid evolution. Yearbook of Physical Anthropology, 44, 54-69.

Varki, A. 2010. Uniquely human evolution of sialic acid genetics and biology. Proceedings of the National Academy of Science, 107, 8939-8946.

Vernot, B., \& Akey, J. M. 2015. Complex history of admixture between modern humans and neandertals. American Journal of Human Genetics, 96, 448-453. doi:10.1016/j.ajhg.2015.01.006

Weaver, T. D., \& Hublin, J. J. 2009. Neandertal birth canal shape and the evolution of human childbirth. Proceedings of the National Academy of Science, 106, 8151-8156.

Wittman, A. B., \& Wall, L. L. 2007. The evolutionary origins of obstructed labor: bipedalism, encephalization, and the human obstetric dilemma. Obstetric and Gynecological Survey, 62, 739-748.

Whitcome, K. K. 2012. Functional implications of variation in lumbar vertebral count among hominins. Journal of Human Evolution, 62, 486-497.

Whitcome, K. K., Shapiro, L. J., \& Lieverman, D. E. 2007. Fetal load and the evolution of lumbar lordosis in bipedal hominins. Nature, 450, 1075-1078.

White, T. D., Asfaw, B., Beyene, Y., Haile-Selassie, Y., Owen Lovejoy, C., Suwa, G., \& WoldeGabriel, G. 2009. Ardipithecus ramidus and the paleobiology of Early Hominids. Science, 326, 75-86. https://doi.org/10.1126/science.1175802

White, T. D., Lovejoy, C. O., Asfaw, B., Carlson, J. P., \& Suwa, G. 2015. Neither chimpanzee nor human, Ardipithecus reveals the surprising ancestry of both. Proceedings of the National Academy of Sciences, 112, 4877-4884. https://doi.org/10.1073/pnas.1403659111

Zindler, R. E. 1978. On the increase of cranial capacity in mankind's lineage: augments and elaborations. Journal of Human Evolution, 7, 295-305. 
Zollikofer, C. P. E., Marcia S., de Leon, P., Lieberman, D. E., Guy, F., Pilbeam, D., et al. 2005. Virtual cranial reconstruction of Sahelanthropus tchadensis.

Nature, 434, 755-759. 
